\title{
Unemployment of Unskilled Labor due to COVID-19 led Restriction on Migration and Trade
}

Biswajit Mandal ( $\sim$ biswajiteco@gmail.com )

Visva-Bharati University

Saswati Chaudhuri

St. Xavier's College

Alaka Shree Prasad

Visva-Bharati University

\section{Research Article}

Keywords: General Equilibrium, COVID-19, Migration, Trade, Unemployment

Posted Date: July 22nd, 2020

DOI: https://doi.org/10.21203/rs.3.rs-45853/v1

License: (c) (1) This work is licensed under a Creative Commons Attribution 4.0 International License.

Read Full License 


\title{
Unemployment of Unskilled Labor due to COVID-19 led Restriction on Migration and Trade
}

\author{
Biswajit Mandal \\ Department of Economics \& Politics, Visva-Bharati University, \\ Santiniketan, India, 731235 \\ Email: biswajiteco@gmail.com \\ Saswati Chaudhuri \\ Department of Economics, St. Xavier's College (Autonomous), \\ Kolkata, India, 700016 \\ Email: sxcsas@sxccal.edu

\begin{abstract}
Alaka Shree Prasad
Department of Economics \& Politics, Visva-Bharati University,

Santiniketan, India, 731235

Email: alakashree56@gmail.com
\end{abstract}

\section{Corresponding Author:}

BISWAJIT MANDAL

Department of Economics \& Politics

Visva-Bharati University

Santiniketan, India

731235

Telephone: (+91) 03463262751-56 Extn. 405

E-mail: biswajiteco@gmail.com / biswajit.mandal@visva-bharati.ac.in 


\title{
Unemployment of Unskilled Labor due to COVID-19 led Restriction on Migration and Trade
}

\begin{abstract}
To combat COVID-19 the entire world has resorted to global lockdown implying restriction on international labor migration and trade. This paper aims to check the effect of such restrictions on the unemployment of unskilled labor in the source country. In competitive general equilibrium framework with three goods and four factors restriction on migration raises unemployment for given factor intensity. The results remain same even in a slightly different structure of the economy. In case of trade restriction, however, the rise or fall in unemployment depends on both the structure of the economy and the factor intensity assumption.

JEL Classification: D5, F22, F12, J6,

Keywords: General Equilibrium, COVID-19, Migration, Trade, Unemployment
\end{abstract}




\title{
Unemployment of Unskilled Labor due to COVID-19 led Restriction on Migration and Trade
}

\begin{abstract}
To combat COVID-19 the entire world has resorted to global lockdown implying restriction on international labor migration and trade. This paper aims to check the effect of such restrictions on the unemployment of unskilled labor in the source country. In competitive general equilibrium framework with three goods and four factors restriction on migration raises unemployment for given factor intensity. The results remain same even in a slightly different structure of the economy. In case of trade restriction, however, the rise or fall in unemployment depends on both the structure of the economy and the factor intensity assumption.

JEL Classification: D5, F22, F12, J6,

Keywords: General Equilibrium, COVID-19, Migration, Trade, Unemployment
\end{abstract}




\section{Introduction and Motivating Environment}

Global outbreak of COVID-19 has unnerved the entire world in last few months. By now it is apparent that Corona virus outbreak took the corridor of physical proximity of affected countries and human beings. This corroborates the role of trade, travel and migration for transmitting Corona virus. And again since the spread of Corona virus has been showing no signs of abating, it has taken the whole world into a synchronized lockdown - complete closure of international borders. A variety of policies are also framed and international sanctions are essentially imposed to restrict international movement of goods and services, and labor. It would lead to inevitable production disruptions. Immobility of labor due to various controls would be accompanied by unnecessary economic cost as well. The impacts would be similar to the one we noticed during H1B visa sanctions imposed by the USA government or during BREXIT. Global production or trade in goods and services would be adversely impacted. When national policies like H1B Visa restriction or BREXIT are responsible for the immobility of labor, a substitute process is also made handy. In this context, production cost rises but the supply chain does not get disrupted because production still takes place (may be by domestic labor). Inability to harness the cost advantage that was possible due to availability of cheap foreign labor will also lead to a rise in the price level along with a fall in demand. This certainly has huge economic impact. However, when the root cause of all restrictions are health related and that too contagious just like the one with COVID-19, then the pathways to an alternative production process no longer remains a viable option. When mobility of resources like labor is restrained 
worldwide, production in a single particular economy does not get affected alone - it affects the global economy at large. Therefore, naturally, possible sufferers of all such policies are worried about the economic upshots in their domestic fronts. In this globalized world, both benefit and ill effects spill over across the globe. And so is the perceived detrimental effects of policies mentioned to combat COVID-19. Thus, finally the issue becomes a political economic one where international relations among different trading countries are persuaded by the policy outcome.

This paper primarily attempts to focus on the effects of restriction on international migration policy which arguably has severe employment effects as this may either cause discontinuation of the stay of immigrants or does not allow new migrant. Such policy would primarily restrict the mobilityof skilled workers such as IT professionals, engineers, etc. And we also know that recent economic prosperity of some countries like Indiahas been predominantly propelled by these skilled workers and their free movement among various occupations and countries. Therefore, the concern is quite justified. What do they apprehend? Due to mobility restriction ${ }^{1}$, domestic supply of skilled workers would rise. This is a kind of reverse migration. Naturally, this would influence prices of other factors and their employment. One possibility is that they get directly employed in the skilled sector either displacing some alreadyemployed skilled youth which moves to other sector and also pushes some unskilled workers towards unemployed pool leading to an increase in unemployment in the economy. Alternatively, skilled workers can make room for some more capital in

\footnotetext{
${ }^{1}$ Quite similar to what happens due to H1B Visa restriction.
} 
the skilled sector and hence return to capital goes up and that of unskilled labor falls. Another possibility is a setback for the existing skilled workers pool. When skilled wage falls, returnto other factors move accordingly. Of course, there are host of other possibilities; we are primarily concerned with the state of unemployment of unskilled workers and unskilled wage where the general apprehension is an increase in unemployment and decrease in wage rate.

Papers like Mayda (2018), Bound et al. (2017), Das et al.(2019), etc. explain the effect of the H1B Visa policy on the host country. However, the existing literature do not provide with much theoretical information or explanation about the effect on employment of the source country. Chaudhuri (2019) is an interesting piece in this line, where the consequences of an anti-immigration policy on the wage gap of the source country has been examined. So, we attempt to fill up the caveat of (un)employment effect in the backdrop of COVID-19.

We first present a competitive general equilibrium model of trade where there are three sectors and four factors. Our structure closely follows Jones $(1965,1971)$. We slightly deviate from these two celebrated models through introduction of unemployment. And for distinction among different labors, unemployment, and their connectedness we follow Agenor and Montiel (2015), Marjit and Acharrya (2003), Marjit and Kar (2011), Acharyya and Kar (2014), Chaudhuri and Mukhopadhyay (2010), Mandal and Mandal (2015), etc. However, we do away with any domestic migration from informal to formal sector, and hence Harris-Todaro migration possibility is not taken into account. We assume three factors - skilled labor, unskilled labor, capital - as 
mobile across the sectors, and one factor as specific to the skilled-labor intensive sector. All the factor are fully employed except unskilled labor. In this backdrop, we find that there is a rise in unemployment of unskilled labors due to therestriction on labor mobility during COVID-19.The resultis found to depend onthe factor intensity assumptions. However, rise or fall in the output of different sectors does not depend on the factor intensity conditions, though the magnitude of change does.

The remaining paper is structured as follows. In the next section, we first propose a competitive trade model and check different prospects. Then we slightly modify the basic model to see how robust is the theoretical predictions of the basic model. We also check how trade control of any sort, which reflects the Corona-driven policy of restriction on international movements of goods, affects the unemployment condition. Finally, we provide some concluding remarks. The relevant mathematical details are, however, relegated to the appendices.

\section{Methodological Background and the Basic Model}

We consider a small open economy endowed with skilled labor $(S)$, unskilled labour $(L)$ and two types of capital (Tand $K)$. There are three sectors, $X, Y$ and $Z$. Both

goods and factor markets are perfectly competitive. $X$ is a skilled-labor $(S)$ intensive sector. Production of $X$ requires $S, K$ and $T ; T$ isspecific to $X$.Equation (1) gives the equilibrium condition of $X$ where per-unit cost is equal to the price:

$$
w_{S} a_{S X}+R a_{T X}+r a_{K X}=P_{X}
$$


Here, $w_{S}$ is the wage of skilled labors; $R$ denotes the rent of $T ; r$ is the rent of $K$; $P_{X}$ is the price of $X$; and $a_{i j} s(i=S, L, K, T ; j=X, Y, Z)$ refer to the input coefficients.

The production of $Y$ requires $S, L$ and $K$, with $K$ being used intensively. $Z$ uses unskilled labor and $K . Y$ canbe taken as a public sector whereas $Z$ is a private sector. The wage of unskilled laboris assumed to differ in both these sectors to capture the fact that employees of the government sector receive more benefit astheir job is relatively secured whencompared to those engaged in the private sector. Thus, the unskilled labors engaged in $Y$ are paid $w_{g}$ whereas those engaged in $Z$ get $\bar{w}\left(w_{g}>\bar{w}\right) \cdot \bar{w}$ is the wage fixed through process ${ }^{2}$ between the employers and the unskilled employees. $Z$ is intensive in the use of $L$. The cost-price equations of $Y$ and $Z$ aregiven,respectively, as follows:

$$
\begin{gathered}
w_{S} a_{S Y}+w_{g} a_{L Y}+r a_{K Y}=P_{Y} \\
\bar{w} a_{L Z}+r a_{K Z}=P_{Z}
\end{gathered}
$$

Here $P_{Y}$ is the price of $Y$ and $P_{Z}$ is the price of $Z$.

The relation between $w_{g}$ and $\bar{w}$,is given as

$$
w_{g}=\bar{w}(1+\tau)
$$

where, $\tau>0$ and exogenously given as a mark up over the private sector wage. Therefore, when private sector wage rises, government sector employees also get higher wage.

\footnotetext{
${ }^{2}$ The mechanism is briefly explained in Appendix A.
} 
We consider $K, T$ and $S$ to be fully employed but full employment of $L$ is not achieved. The endowment conditions for unskilled labor, skilled labor, $T$ and $K$ areas follows:

$$
\begin{gathered}
a_{L Y} Y+a_{L Z} Z=\bar{L}-L_{u} \\
a_{S X} X+a_{S Y} Y=\bar{S} \\
a_{T X} X=\bar{T} \\
a_{K X} X+a_{K Y} Y+a_{K Z} Z=\bar{K}
\end{gathered}
$$

$L_{u}$ in equation (5) denotes the amount of unemployed unskilled labors in the economy. The amount of unskilled labors who are employed is simply given as:

$$
L_{E}=\bar{L}-L_{u}
$$

This completes the framework of our model. We have nineequations and nine variables namely, $w_{S}, w_{g}, r, R, X, Y, Z, L_{u}$ and $L_{E} \cdot w_{g}$ is obtained from (4). $r$ is determined from (3) as $\bar{w}$ is given. With rand $w_{g}$ at hand, we get the value of $w_{S}$ from (2). Putting the value of $w_{S}$ and $r$ in (1), we get $R . a_{i j} s(i=L, S, K, T ; j=X, Y, Z)$ are determined by the Constant Returns to Scale (CRS) assumption. Xis calculated from (7). Using the value of $X$ in (6) we calculate the value of $Y$. By substituting the value of $X$ and $Y$ in (8), $Z$ is determined. Further, substituting the value of $Y$ and $Z$ in (5) we get $L_{u}$.Putting the value of $L_{u}$ in (9), we get the value of $L_{E}$. Thus, the model is solved.

\subsection{Restriction international labor mobility}

With a restriction oninternationallabor movement migration of particularly skilled laborsfrom developing or poor countries to rich countries is stopped and/or 
skilled labors are forced to return from developed to developing economies. As a result, there is an increase in the supply of skilled laborin the source economy. Since there is no change in price of the goods, there will be no change in the factor prices whatsoever and consequently the input coefficients remain unchanged.However, there may be changes in the output of different sectors. From (7), we get the percentage change in $X$ as,

$$
\widehat{X}=(-) \hat{a}_{T X}=0
$$

From equation (10), we observe that the percentage change in $X$ is zero as non-changing factor prices constrained the substitution possibility. Thus, in spite of being $S$ intensive, with the increase in the supply of skilled labor because of COVID-19, $X$ remains unaffected. Fixity of $T$ could be another complementary reason for such result. Consequently, $X$ producers have no further incentive to change their output level as, given the situation, they are already producing at their optimal point.

Now, using (10), from (6) we have the percentage change in the output of sector $Y$ as

$$
\hat{Y}=\frac{\hat{S}}{\lambda_{S Y}}>0 \text { as } \hat{S}>0
$$

Using the values of $\hat{X}$ and $\hat{Y}$, from (8) we getthe relative change in $Z$ :

$$
\hat{Z}=(-) \frac{\lambda_{K Y}}{\lambda_{K Z} \lambda_{S Y}} \hat{S}<0 \text { ass } \hat{S}>0
$$

The positive change in $Y$, as seen in equation (11), is because sector $X$ does not absorb the skilled laborsreturned to the economy. Therefore, the excess skilled labor is employed in $Y$. Since $Y$ also uses $L$, who are paid more than those engaged in $Z$, unskilled labors from $Z$ will move out to get employed in $Y$. As a result, $Z$ contracts. When $Z$ 
contracts, along with $L$ it also releases $K$ which can be usedin $Y$. Thus, there is an expansion of $Y$ as, unlike $X$, it does not face any input constraints. Therefore, with a restriction on migration, $X$ remains unchanged, sector $Y$ expands and sector $Z$ contracts. Note that this result is irrespective of the factor intensity conditions.

Now, when $Y$ expands and $Z$ contracts, $Z$ being unskilled-labour intensive releases more unskilled labors than required in $Y$. Therefore, there will be a rise in unemployment level of unskilled labors. To check the mathematical effect of international lockdown related restriction on labor mobility on the employment of unskilled labor mathematically, we use equation (5) and with the help of equations (9), (11) and (12) we obtainthe change in employment of unskilled labor as follows:

$$
\hat{L}_{E}=\frac{\hat{S}}{\lambda_{S Y} \lambda_{K Z}}\left(\lambda_{K Z} \lambda_{L_{E} Y}-\lambda_{L_{E} Z} \lambda_{K Y}\right)<0
$$

Or, the change in unemployment level of unskilled labor is

$$
\hat{L}_{U}=(-) \frac{\lambda_{L_{E} L}}{\lambda_{L_{U} L} \lambda_{S Y} \lambda_{K Z}}\left(\lambda_{K Z} \lambda_{L_{E} Y}-\lambda_{L_{E} Z} \lambda_{K Y}\right) \hat{S}>0
$$

As presumed, since $Y$ is $K$ intensive and $Z$ is $L$ intensive, from equation (13), there is a fall in the employment of unskilled labor or from (14) a rise in the unemployment level of unskilled labor.Thus, we have the following proposition:

Proposition 1: Given the factor intensity assumptions, there is a rise in the unemployment level of unskilled labors because ofinternational lockdown during COVID-19. 


\section{A Slightly Different Framework}

In this section, we develop a variant of the model considered in the previous section. Here the skilled-labor intensive sector $(X)$ uses only skilled labor and $K$ for production. $Y$ can be regarded as the government manufacturing sector which uses $S, L$ and $K$. Asin the previous model, the unskilled labors employed in $Y$ receive higher wagethan those employed in non-government sectors. To capture this we assume the wage rate in $Y$ is $w_{g}$ which is higher than $\bar{w}$ - the wage rate in sector $Z$.Here, we assume $T$ to be a type of capital that is specific to $Z$. Therefore, sector $Z$ uses $L$ and a specific factor $T$ for production. The conditions showing the cost-price equality of $X, Y$ and $Z$ are given below:

$$
\begin{gathered}
w_{S} a_{S X}+r a_{K X}=P_{X} \\
w_{S} a_{S Y}+w_{g} a_{L Y}+r a_{K Y}=P_{Y} \\
\bar{w} a_{L Z}+R a_{T Z}=P_{Z}
\end{gathered}
$$

We assume full employment of $K, T$, and existing $S$, in the economy. A part of unskilled labor is, however, unemployed. The endowment equations of the factors are:

$$
\begin{gathered}
a_{S X} X+a_{S Y} Y=\bar{S} \\
a_{L Y} Y+a_{L Z} Z=L-L_{u}=L_{E} \\
a_{T Z} Z=\bar{T} \\
a_{K X} X+a_{K Y} Y=\bar{K}
\end{gathered}
$$

Equations (15)-(21) along with equations(4) and (9), represent the basic structure of the model. There are nine equations and nine variables $\left(w_{S}, w_{g}, r, R, X, Y, Z, L_{u}\right.$ and $\left.L_{E}\right)$. Hence, the model is solvable. 
With the given structure of the economy, if there is an increase in the supply of skilled labor due to lockdown, the following changes occur in each of the sectors:

$$
\begin{gathered}
\hat{X}=(-) \frac{\lambda_{K Y}}{\lambda_{K X} \lambda_{S Y}-\lambda_{K Y} \lambda_{S X}} \hat{S}>0 \\
\hat{Y}=\frac{\lambda_{K X}}{\lambda_{K X} \lambda_{S Y}-\lambda_{K Y} \lambda_{S X}} \hat{S}<0 \\
\hat{Z}=0
\end{gathered}
$$

The change in employment of unskilled labors is

$$
\hat{L}_{E}=\frac{\lambda_{K X} \lambda_{L_{E} Y}}{\lambda_{K X} \lambda_{S Y}-\lambda_{K Y} \lambda_{S X}} \hat{S}<0
$$

Or, the change in unemployment of unskilled labor is

$$
\hat{L}_{U}=(-) \frac{\lambda_{L_{E} L} \lambda_{K X} \lambda_{L_{E} Y}}{\lambda_{L_{U} L}\left(\lambda_{K X} \lambda_{S Y}-\lambda_{K Y} \lambda_{S X}\right)} \hat{S}>0
$$

As in the previous model, there is no change in the price of any good so the factor prices and input coefficients remain unchanged.Now, as the skilled-labor intensive sector uses only skilled labor and $K$, the inflow of skilled labor will positively affect the size of sector $X$ (equation (22)). Non specificity of any factor in $\mathrm{X}$ is doing the trick. As $X$ also uses $K$, additional amount of $K$ is drawn from $Y$ and thus there is a fall in $Y$ (equation (23)). $Y$ releases capital $(K)$, skilled labor and unskilled labor. Kand $S$ get employed in $X$ but $L$ remains unemployed. Though $Z$ uses $L$ for production but the limited supply of $T$ and unchanged factor prices restrict $Z$ from changing the input composition, leaving $Z$ unaffected because of restriction on international labor mobility (equation (24)). Therefore, there is excess supply of unskilled labor leading to a rise in 
unemployment (equations(25) and (26))of unskilled workers. However, these results depend on the factor intensity assumption.If $X$ was $K$ intensive and $Y$ was skilled-labor intensive then $Y$ would absorb the excess supply of skilled labor and $X$ would contract in order to supply $K$ to $Y$. As $Y$ also uses $L$, the employment level of unskilled labor would have risen.Therefore, when we consider a different framework, factor intensity in production plays an important role in determining the effect of an increase in the supply of skilled labors on the employment of unskilled factors. Thus, we have,

Proposition 2:Not the structure of the economy, but the factor intensity of different sectors is fundamental in determining the level of unemployment of unskilled labordue to restriction on international labor mobility during COVID-19.

Together with this, we also propose:

Proposition 3: If a particular sector faces any resource constraint, it remains unaffected due tointernational lockdown.

Proof:In the basic model,one specific factor, whose supply islimited, was used by the skilled-labor intensive sector. Even if the skilled labor is used intensively in that sector, unavailability ofthe specific input in the economy restricted it from expanding. In contrast to this, in the extended model when $X$ was able to acquire inputs, we saw its expansion with increased supply of skilled labor.On the other hand, in the extended model, sector $Z$ was unaffected because of the similar reason that led to non-changing $X$ in the basic model.

\section{Restriction on Trade and Unemployment of Unskilled Labors}


In the basic model, suppose countries impose restriction on its imports. If $X$ is the importable good, there must be a decline in the demand for $X$ from the rest of the world (ROW) and since the country concerned is assumed as small compare to the ROW, the price of $X$ would decline. A fall in the price of $X$ will cause its output to fall releasing some of the factors employed in it. AsT is specific to $X$, the rent of $T, R$ fallsand $T$ would be used more intensively in $X$. On the other hand, assuming no change in the requirement of $K$, there will be a fall in per-unit requirement of $S$ (though the factor intensity assumption would not be changed). Thus, $T$ remains fully employed in $X$ but some $K$ and $S$ are released ${ }^{3}$. Sand $K$ gets employed in $Y$. Since $Y$ is $K$ intensive it requires more $K$ than released from $X$. This extra demand for $K$ is met by a fall in $Z$. When $Z$ falls, $L$ is also released which is used in $Y$. However, as $Z$ is unskilled labor intensive more $L$ is released than demanded for the expansion of $Y$ leading to an increase in unemployment of unskilled labors4.

Now if we consider the extended version of the basic model, restriction in imports by the ROW again leads to a fall in the price of $X$ discouraging its production. There is a reduction in the output of $X$ and consequently $S$ and $K$ are released. These $S$ and $K$ can get employed in $Y$. Also, following Stolper-Samuelson's argument there is a fall in the price of the factor used intensively in $X$ i.e., $w_{S}$, and there is a rise in the price of less intensively used factor i.e., a rise in $r$. In what follows, there will be substitution of

\footnotetext{
${ }^{3}$ Here, except $R$, all the factor prices remain unchanged. As $\bar{w}$ is fixed, from equation (3)rdoes not change and from equation (4) $w_{g}$ remains constant. Moving to equation (2), since $P_{Y}$ is unaltered, $w_{S}$ also remains the same. Thus, only $R$ is affected by trade restriction.

${ }^{4}$ Mathematical details are given in Appendix B.
} 
factors in production. Again, as $Y$ also requires $L$, additional $L$ is acquired from the unemployed pool of the economy thereby reducing the level of unemployment. ${ }^{5}$ Sector $Z$ remains unaltered as it uses a specific factor, $T$.

Therefore, unlike the case of restriction on international labor migration, when the ROW opts for restriction on trade, the effect on unemployment of unskilled labors depends on the specific structure of the economy.

Thus, we propose that:

Proposition 4:Unlike restriction on labor migration, the effect of trade restriction on unskilled unemployment depends on specific structure of the economy.

Proof: See discussion above.

\section{Conclusions}

In this paper, we consider two slightly different general equilibrium frameworks to check the effect ofinternational lockdown which has been adopted by all countries across the globe to control the spread of COVID-19. First we analyze the case of restrictionon labor migration on employment of unskilled labor. Though having different structures of the economy, we see that unemployment of unskilled labor rises in both the frameworks. But the factor intensity assumptionis important irrespective of specific economic setup. In the next section, we check the employment effect of another protectionist policy, trade restriction. It is found that in case of restriction on international trade, the effect on unemployment depends on the type of economy;

\footnotetext{
${ }^{5}$ Mathematical calculations are provided in Appendix C.
} 
though as in the case migration control, the factor intensity here also plays an important role in arriving at the results.

\section{Appendices}

\section{Appendix A}

\section{Determination of wage in sector $Z$}

The wage is determined by the labor unions by maximizing their utility function subject to the labor demand. We assume the firms to be price takers. Let the utility function of the union be: $U=U\left(w, L_{Z}, p_{Z}\right)$; where $w i \mathrm{~s}$ the wage rate, $L_{Z}$ is the employment in $Z, p_{Z}$ is the relative price of $Z$. $U$ is quasi-concave and increasing in $w$ and $L_{Z}$. 
The labor demand function is $L_{Z}=Z c_{w}=L_{Z}(w, r)$, here $r$ is the rent of $K, c_{w}$ is the partial derivative of the cost function, $c(w, r)$, and $\mathrm{Z}$ is the output. The demand for labor is negatively related to wage.

The first order condition implies $\frac{\delta u / \delta w}{\delta u / \delta L_{Z}}=-\frac{\delta L_{Z}}{\delta w}$

$\Rightarrow M R S_{w, L_{Z}}=$ Slope of the labor demand curve

The wage rate is determined from the above condition. The second order condition of the maximization problem also holds:

$$
\frac{\delta^{2} u}{\delta w^{2}}+2 \frac{\delta^{2} u}{\delta w \delta L_{Z}} \frac{\delta L_{Z}}{\delta w}+\frac{\delta^{2} u}{\delta L_{Z}{ }^{2}}\left(\frac{\delta L_{Z}}{\delta w}\right)^{2}+\frac{\delta u}{\delta L_{Z}} \frac{\delta^{2} L_{Z}}{\delta w^{2}}<0
$$

\section{Appendix B}

Expressing relative change by “^" notation, from equations (1)-(3) we get,

$$
\begin{gathered}
\widehat{w}_{S} \theta_{S X}+\widehat{R} \theta_{T X}+\hat{r} \theta_{K X}=\widehat{P}_{X} \\
\widehat{w}_{S} \theta_{S Y}+\hat{r} \theta_{K Y}=0 \\
\hat{r}=0
\end{gathered}
$$

Putting the value of $\hat{r}$ in (B.2), $\widehat{w}_{S}=0$. Following this, from (B.1), $\hat{R}=\frac{\hat{P}_{X}}{\theta_{T X}}$.

As $\widehat{w}_{S}=\hat{r}=0$, the input coefficients of $Y$ and $Z$ remains unchanged. However, because of change in $R$, there will be changes in factor requirements of $X$. For simplicity, we assume $\hat{a}_{K X}=0$. Then, using the Envelope condition and the elasticity of substitution we get, $\hat{a}_{S X}=\frac{\sigma_{X}}{\left(1-\theta_{K X}\right)} \hat{P}_{X}<0$ and $\hat{a}_{T X}=-\frac{\sigma_{X} \theta_{S X}}{\left(1-\theta_{K X}\right) \theta_{T X}} \hat{P}_{X}>0$

Using (5)-(8) we have 


$$
\begin{gathered}
\lambda_{L_{E} Y} \hat{Y}+\lambda_{L_{E} Z} \hat{Z}=\hat{L}_{E} \\
\lambda_{S X} \hat{X}+\lambda_{S X} \hat{a}_{S X}+\lambda_{S Y} \hat{Y}=0 \\
\hat{X}=-\hat{a}_{T X}<0 \\
\lambda_{K X} \hat{X}+\lambda_{K Y} \hat{Y}+\lambda_{K Z} \hat{Z}=0
\end{gathered}
$$

Substituting the value of $\hat{X}$ in (B.5)

$$
\hat{Y}=-\frac{\lambda_{S X}}{\lambda_{S Y}}\left(\widehat{a}_{S X}-\hat{a}_{T X}\right) \Rightarrow \hat{Y}=-\frac{\lambda_{S X}}{\lambda_{S Y}} \frac{\sigma_{X} \hat{P}_{X}}{\theta_{T X}}>0
$$

Using the value of $\hat{X}$ and $\hat{Y}$ in (B.7)

$$
\begin{gathered}
\hat{Z}=\frac{\lambda_{K X}}{\lambda_{K Z}} \hat{a}_{T X}-\frac{\lambda_{K Y}}{\lambda_{K Z}}\left\{-\frac{\lambda_{S X}}{\lambda_{S Y}}\left(\widehat{a}_{S X}-\hat{a}_{T X}\right)\right\} \\
\Rightarrow \hat{Z}=\frac{\sigma_{X} \hat{P}_{X}}{\theta_{T X} \lambda_{S Y} \lambda_{K Z}}\left\{\theta_{S X}\left(\lambda_{S X} \lambda_{K Y}-\lambda_{S Y} \lambda_{K X}\right)+\lambda_{K Y} \lambda_{S Y} \theta_{T X}\right\}
\end{gathered}
$$

Here, following the factor intensity assumptions of the model, $\left(\lambda_{S X} \lambda_{K Y}-\lambda_{S Y} \lambda_{K X}\right)>0$.

Therefore, as $\hat{P}_{X}<0, \hat{Z}<0$.

From (B.4),

$$
\hat{L}_{E}=-\frac{\sigma_{X} \hat{P}_{X}}{\theta_{T X} \lambda_{S Y} \lambda_{K Z}\left(1-\theta_{K X}\right)}\left\{\left(\lambda_{L_{E} Y} \lambda_{K Z}-\lambda_{L_{E} Z} \lambda_{K Y}\right) \lambda_{S X}\left(1-\theta_{K X}\right)+\lambda_{L_{E} Z} \lambda_{K X} \theta_{S X} \lambda_{S Y}\right\}<0
$$

Since $Z$ is $L$ intensive and $Y$ is $K$ intensive, a fall in $P_{X}$ reduces the level of employment of unskilled labors.

\section{Appendix C}

From equations (15)-(17) we get, 


$$
\begin{gathered}
\widehat{w}_{S} \theta_{S X}+\hat{r} \theta_{K X}=\hat{P}_{X} \\
\widehat{w}_{S} \theta_{S Y}+\hat{r} \theta_{K Y}=0 \\
\hat{R}=0
\end{gathered}
$$

From (C.2), $\hat{r}=-\widehat{w}_{S} \frac{\theta_{S Y}}{\theta_{K Y}}$. Putting this in (C.1) we get, $\widehat{w}_{S}=\left(\frac{\theta_{K Y}}{\theta_{S X} \theta_{K Y}-\theta_{S Y} \theta_{K X}}\right) \hat{P}_{X}<0$, as $\widehat{P}_{X}<0$, and $X$ and $Y$ are $S$ and $K$ intensive, respectively. Using the obtained value of $\widehat{w}_{S}$, we calculate $\hat{r}=-\frac{\theta_{S Y}}{\theta_{S X} \theta_{K Y}-\theta_{S Y} \theta_{K X}} \hat{P}_{X}>0$

With changes in the $w_{S}$ and $r$ there are changes in the per unit factor requirements of $X$ and $Y$. Using the Envelope condition and the elasticity of substitution between $S$ and $\operatorname{Kin} X, \sigma_{X}$, we get

$$
\begin{gathered}
\hat{a}_{S X}=-\frac{\theta_{K X}\left(1-\theta_{L Y}\right) \sigma_{X}}{\theta_{S X} \theta_{K Y}-\theta_{S Y} \theta_{K X}} \hat{P}_{X}>0 \\
\hat{a}_{K X}=\frac{\theta_{S X}\left(1-\theta_{L Y}\right) \sigma_{X}}{\theta_{S X} \theta_{K Y}-\theta_{S Y} \theta_{K X}} \hat{P}_{X}<0
\end{gathered}
$$

Similarly, assuming $\hat{a}_{L Y}=0$, we obtain

$$
\begin{gathered}
\hat{a}_{S Y}=-\frac{\theta_{K Y} \sigma_{Y}}{\theta_{S X} \theta_{K Y}-\theta_{S Y} \theta_{K X}} \hat{P}_{X}>0 \\
\hat{a}_{K Y}=\frac{\theta_{S Y} \sigma_{Y}}{\theta_{S X} \theta_{K Y}-\theta_{S Y} \theta_{K X}} \hat{P}_{X}<0
\end{gathered}
$$

Following this, using equations (18)-(21)

$$
\begin{gathered}
\lambda_{S X} \hat{X}+\lambda_{S Y} \hat{Y}+\lambda_{S X} \hat{a}_{S X}+\lambda_{S Y} \hat{a}_{S Y}=0 \\
\lambda_{L_{E} Y} \hat{Y}+\lambda_{L_{E} Z} \hat{Z}=\widehat{L}_{E} \\
\hat{Z}=0
\end{gathered}
$$




$$
\lambda_{K X} \hat{X}+\lambda_{K Y} \hat{Y}+\lambda_{K X} \widehat{a}_{K X}+\lambda_{K Y} \widehat{a}_{K Y}=0
$$

Using Cramer's rule, from (C.4) and (C.7) we get the following values

$$
\begin{gathered}
\hat{X}=\frac{\lambda_{S Y}\left(\lambda_{K X} \hat{a}_{K X}+\lambda_{K Y} \hat{a}_{K Y}\right)-\lambda_{K Y}\left(\lambda_{S X} \hat{a}_{S X}+\lambda_{S Y} \hat{a}_{S Y}\right)}{\lambda_{S X} \lambda_{K Y}-\lambda_{S Y} \lambda_{K X}}<0 \\
\hat{Y}=\frac{\lambda_{K X}\left(\lambda_{S X} \hat{a}_{S X}+\lambda_{S Y} \hat{a}_{S Y}\right)-\lambda_{S X}\left(\lambda_{K X} \hat{a}_{K X}+\lambda_{K Y} \hat{a}_{K Y}\right)}{\lambda_{S X} \lambda_{K Y}-\lambda_{S Y} \lambda_{K X}}>0
\end{gathered}
$$

From (C.5), $\hat{L}_{E}=\lambda_{L_{E} Y} \hat{Y}$

$$
\Rightarrow \hat{L}_{E}=\frac{\lambda_{L_{E} Y}\left\{\lambda_{K X}\left(\lambda_{S X} \hat{a}_{S X}+\lambda_{S Y} \hat{a}_{S Y}\right)-\lambda_{S X}\left(\lambda_{K X} \hat{a}_{K X}+\lambda_{K Y} \hat{a}_{K Y}\right)\right\}}{\lambda_{S X} \lambda_{K Y}-\lambda_{S Y} \lambda_{K X}}>0
$$

NOTE: There is no conflict of interest.

\section{References}

Acharyya, R., \& Kar, S. (2014). International trade and economic development. Oxford ; New York: Oxford University Press.

Agénor, P. R., \& Montiel, P. J. (2015). Development macroeconomics. Princeton university press.

Bound, J., Khanna, G., \& Morales, N. (2017). Understanding the Economic Impact of the H1B Program on the US (No. w23153). National Bureau of Economic Research. 
Chaudhuri, S. (2019). Anti-immigration policy in the destination country and skilledunskilled wage inequality in a source economy with or without unemployment. Economics \& Politics, 31(3), 465-484.

Chaudhuri, S., \&Mukhopadhyay, U. (2010). Revisiting the informal sector: A general equilibrium approach. New York: Springer.

Das, G., Marjit, S., \&Kar, M. (2019). Skill, innovation and wage inequality: Can immigrants be the trump card? CESifo Working Paper No. 7794.

Jones, R. W. (1965). The structure of simple general equilibrium models. The Journal of Political Economy, 73 (6), 557-572.

Jones, R. W. (1971). A three-factor model in theory, trade and history. In J. Bhagwati, R. W. Jones, R. A. Mundell, \& J. Vanek (Eds.), Trade, Balance of Payments and Growth (pp. 321). Amsterdam, North-Holland.

Mandal, B., \& Mandal, A. (2015). A note on how and why growth and unemployment go hand in hand in developing economies. International Economic Journal, 29(4), 681-693. Marjit, S., \&Acharyya, R. (2003). International trade, wage inequality and the developing economy: A general equilibrium approach. Heidelberg; New York: Physica-Verlag.

Marjit, S., \& Kar, S. (2011). The outsiders: Economic reform and informal labour in a developing economy. New Delhi: Oxford University Press.

Mayda, A. M., Ortega, F., Peri, G., Shih, K., \&Sparber, C. (2018). The effect of the H-1B quota on the employment and selection of foreign-born labor. European Economic Review, $108,105-128$. 
